\title{
Dark feature in EUV post-flare loops
}

\section{Qiao Song ${ }^{1,2,3}$, Mei Zhang ${ }^{3}$, Jing-Song Wang ${ }^{1}$, Xue-Shang Feng ${ }^{2}$ and Xiao-Xin Zhang ${ }^{1}$}

\footnotetext{
${ }^{1}$ Key Laboratory of Space Weather, National Center for Space Weather, China Meteorological Administration, Beijing 100081, China. email: qsong@nao.cas.cn

${ }^{2}$ State Key Laboratory of Space Weather, National Space Science Center, Chinese Academy of Sciences, Beijing 100190, China.

${ }^{3}$ Key Laboratory of Solar Activity, National Astronomical Observatories, Chinese Academy of Sciences, Beijing 100012, China.
}

\begin{abstract}
Post-flare loops (PFLs) usually appear in the late phase of eruptive flares as an arcade-like loop system. The Atmospheric Imaging Assembly (AIA) on-board the Solar Dynamics Observatory (SDO) delivers continuously high temporal and spatial resolution extreme ultraviolet (EUV) observations, providing a unique chance to study the PFLs. In this work, we use $S D O /$ AIA high-quality EUV images to study the dark loop-like features in post-flare loops (DPFLs) of an X5.4 flare. Our analysis shows that: 1) the DPFLs are darker than their surrounding and the bright loops, but are brighter than the EUV background; 2) the DPFLs appear in multiple EUV channels, which indicates that they are absorption features; 3) the DPFLs are associated with downflows that are caused by the thermal instability in the cooling process of the flare.
\end{abstract}

Keywords. Solar flare, Post-flare loop

\section{Introduction}

Post-flare loops (PFLs) usually appear in the late phase of eruptive flares as an arcadelike loop system. PFLs were first found in $\mathrm{H} \alpha$ observations in 1960s and then in other wavelengths (Bruzek 1964; Sheeley et al. 1975; Kahler 1977). According to the standard flare model, PFLs are a surface phenomenon presented in the cooling process of a flare (Forbes \& Acton 1996; Priest \& Forbes 2002). The twisted magnetic field in active regions provides tremendous energies to heat the plasmas during the flares (Schrijver et al. 2008; Song et al. 2013). When these plasmas get cooling down in the corona, the PFLs appear to grow in a particular wavelength, such as $\mathrm{H} \alpha$, extreme ultraviolet (EUV), and X-ray.

The Atmospheric Imaging Assembly (AIA, Lemen et al. 2012) on-board the Solar Dynamics Observatory (SDO, Pesnell et al. 2012) opens a new window for EUV observations of PFLs, especially for dark features that deserve further studies. This work focuses on the dark loop-like features of PFLs in SDO/AIA EUV images, which are called dark post-flare loops (DPFLs) hereafter. In the next sections, we first outline the data used in this work in Section 2, then present the results in Section 3, and a brief summary is given in Section 4.

\section{Data}

This work uses high-cadence (12 s) and high-resolution $\left(0.6^{\prime \prime}\right.$ pixel $\left.^{-1}\right)$ EUV observations of NOAA active region (AR) 11429 obtained by SDO/AIA on 2012 March 7. AR 

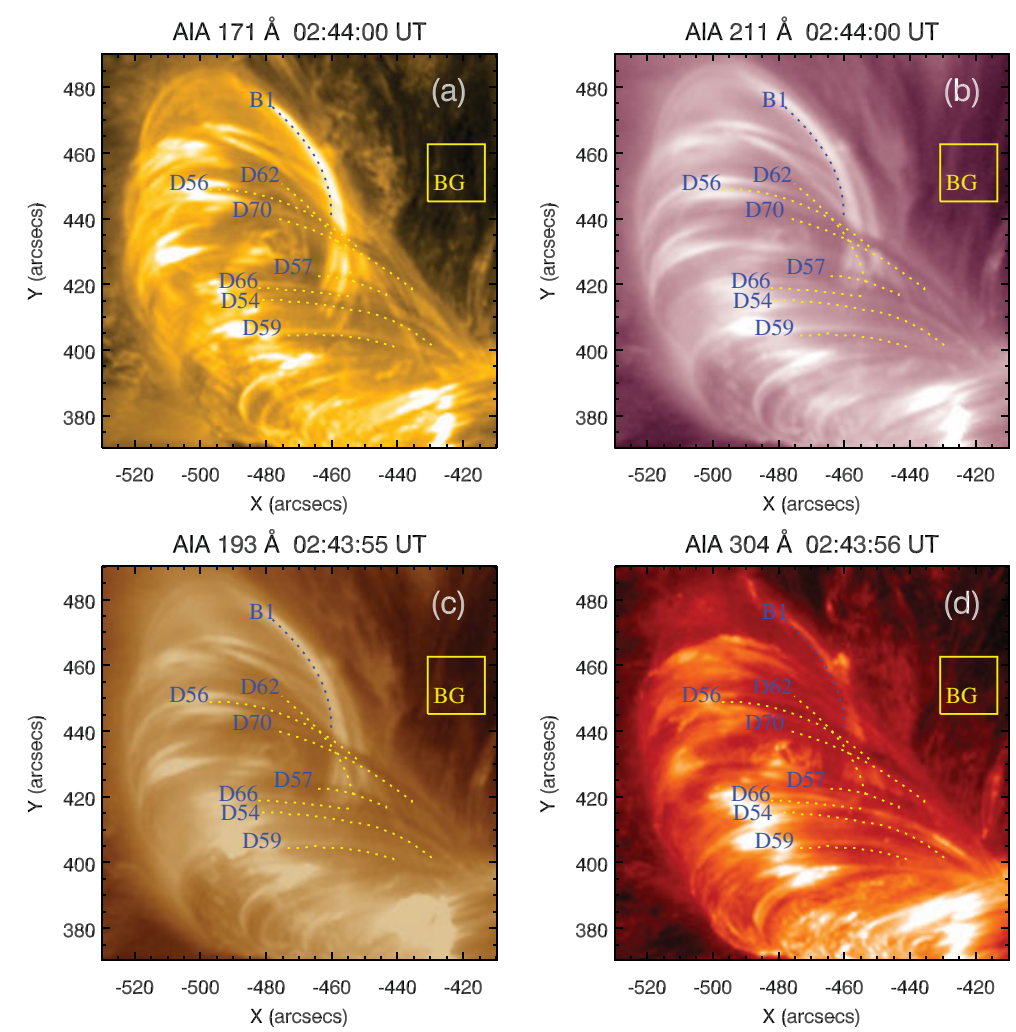

Figure 1. SDO/AIA multiband EUV observations of dark post-flare loops (DPFLs) in the active region NOAA 11429 around 02:44 UT on 2012 March 7.

11429 was on the solar disk that day, and it is convenient to use SDO/HMI longitudinal magnetograms as a reference. We run standard routines, such as aia_prep.pro and hmi_prep.pro, in the Solar SoftWare (SSW) to convert level 1 data to level 1.5 and correct the solar rotation.

\section{Results}

An X5.4 flare erupted from AR 11429 at 00:02 UT on 2012 March 7. About 40 minutes later, the first DPFL appeared in the post-flare loops of the flare. These dark loop-like features could be found in multiple SDO/AIA channels. However, the DPFLs appear sharper and clearer in the $171 \AA$ channel, and we use this channel to identify them one by one for a period of about 4 hours. Figure 1 presents (by yellow dotted lines) seven of these DPFLs in $171 \AA, 211 \AA, 193 \AA$, and $304 \AA$ channels. The DPFLs are darker than their surrounding and the bright loops such as B1, but are brighter than the background (BG). It indicates that the DPFLs were absorption features.

The evolution of a DPFL is displayed in Figure 2. It formed near the top of a loop around 02:14 UT, then grew to the footpoint of the loop, and finally disappeared around 02:44 UT. Its lifetime was about 30 minutes, which is longer than the average lifetime of DPFLs. The moving features along the loop suggest that they were downflows from the loop top to the loop footpoint. Calculations suggest that these downflows are condensations caused by thermal instability in the cooling process of the flare. 


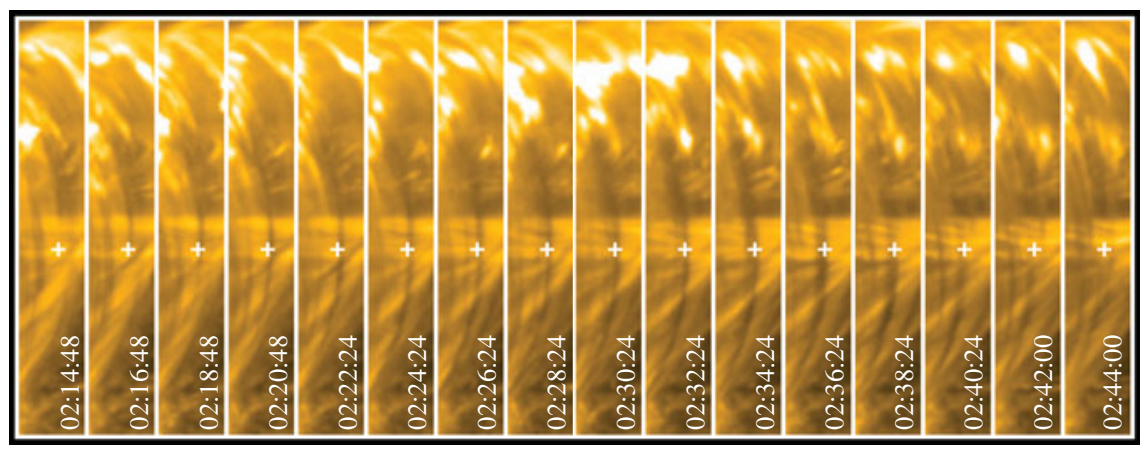

Figure 2. Evolution of a DPFL in SDO/AIA $171 \AA$ images.

\section{Summary}

We use $S D O /$ AIA high-quality EUV images to study dark loop-like features in postflare loops (DPFLs) of an X5.4 flare. Following results are obtained: 1) the DPFLs are darker than their surrounding and the bright loops, but are brighter than the EUV background; 2) the DPFLs are found in multiple EUV channels, which indicates that they are absorption features; 3 ) the DPFLs are related to the downflows that are caused by thermal instability in the cooling process of the flare. The absorption in DPFLs may be caused by hydrogen and helium in the cooling process, which was found in previous work made by SOHO/EIT and TRACE (Anzer \& Heinzel 2005). More examples and analysis are undertaking and will be presented in a future paper.

\section{Acknowledgements}

The authors sincerely thank Prof. Petr Heinzel for his helpful comments. We gratefully thank Prof. J. Zhang, Dr. Y. Z. Zhang, and Dr. G. P. Zhou for inspiring the original idea and giving many useful advices to this work. The work is supported by the National Hightech R\&D Program of China (2012AA121000), the National Basic Research Program of China (2011CB811403), the National Natural Science Foundation of China (41404136, 40931056, 11125314, 11221063 and 11373004).

\section{References}

Anzer, U. \& Heinzel, P. 2005, ApJ, 622, 714

Bruzek, A. 1964, ApJ, 140, 746

Forbes, T. G. \& Acton, L. W. 1996, ApJ, 459, 330

Kahler, S. 1977, ApJ, 214, 891

Lemen, J. R., Title, A. M., \& Akin, D. J., et al. 2012, Solar Physics, 275, 17

Pesnell, W. D., Thompson, B. J., \& Chamberlin, P. C. 2012, Solar Physics, 275, 3

Priest, E. R. \& Forbes, T. G. 2002, A\&A Rev., 10, 313

Schrijver, C. J., De Rosa, M. L., \& Metcalf, T., et al. 2008, ApJ, 675, 1637

Sheeley, N. R., Jr., Bohlin, J. D., \& Brueckner, G. E., et al. 1975, Solar Physics, 45, 377

Song, Q., Zhang, J., Yang, S. H., \& Liu, Y. 2013, Research in Astronomy and Astrophysics, 13, 226 